\title{
A new measurement of the spin-dependent structure function $g_{1}(x)$ of the deuteron
}

\author{
Spin Muon Collaboration (SMC)
}

\begin{abstract}
D. Adams ${ }^{q}$, B. Adeva ${ }^{\text {s }}$, E. Arik ${ }^{b}$, A. Arvidson ${ }^{v}$, B. Badelek ${ }^{v, x}$, M.K. Ballintijn ${ }^{n, 1}$,
\end{abstract} G. Bardin ${ }^{r}$, G. Baum ${ }^{\text {a }}$, P. Berglund ${ }^{\text {g }}$, L. Betev ${ }^{\ell}$, R. Birsa ${ }^{\text {u }}$, P. Björkholm ${ }^{\mathrm{v}}$, B.E. Bonner ${ }^{\mathrm{q}}$, N. de Botton ${ }^{r}$, F. Bradamante ", A. Bressan ", S. Bültmann ${ }^{\mathrm{a}}$, E. Burtin ${ }^{\mathrm{r}}$, C. Cavata ${ }^{\mathrm{r}}$, M. Clocchiatti " , D. Crabb ${ }^{\text {w }}$, J. Cranshaw ${ }^{q, 2}$, T. Çuhadar ${ }^{b}$, S. Dalla Torre ${ }^{\text {, }}$, R. van Dantzig ${ }^{n}$, A. Deshpande ${ }^{y}$, S. Dhawan ${ }^{y}$, C. Dulya ${ }^{c}$, A. Dyring ${ }^{v}$, S. Eichblatt ${ }^{q}$, D. Fasching ${ }^{p}$, F. Feinstein ${ }^{\text {r }}$, C. Fernandez ${ }^{\text {s,h }}$, B. Frois ${ }^{\text {d, }}$, J.A. Garzon ${ }^{\text {s,h }}$, M. Giorgi ${ }^{\text {u, }}$ E. von Goeler ${ }^{\circ}$, G. Gracia ${ }^{\mathrm{s}}$, R. Granier de Cassagnac ${ }^{\mathrm{r}}, \mathrm{N}$. de Groot $^{\mathrm{n}}$,

M. Grosse Perdekamp ${ }^{c}$, E. Gülmez ${ }^{\text {b }}$, D. von Harrach ${ }^{j}$, T. Hasegawa ${ }^{m, 3}$, P. Hautle ${ }^{\text {d,4 }}$, N. Hayashi ${ }^{m, 5}$, C.A. Heusch ${ }^{6}$, N. Horikawa ${ }^{m}$, V.W. Hughes ${ }^{y}$, G. Igo $^{c}$, S. Ishimoto ${ }^{\mathrm{m}, 7}$, T. Iwata ${ }^{\mathrm{m}}$, E.M. Kabuß ${ }^{j}$, T. Kageya ${ }^{\mathrm{m}}$, A. Karev ${ }^{i}$, H.J. Kessler ${ }^{\text {e }}$, T.J. Ketel ${ }^{\mathrm{n}}$, A. Kishi ${ }^{\mathrm{m}}$, Yu. Kisselev ${ }^{i}$, L. Klostermann ${ }^{\text {n, D. Krämer }}{ }^{\text {a }}$, V. Krivokhijine ${ }^{\mathrm{i}}$, W. Kröger ${ }^{\mathrm{d}, 6}, \mathrm{~K}$, Kurek $^{\mathrm{x}}$, J. Kyynäräinen $^{\mathrm{d}, \mathrm{g}}$, M. Lamanna ${ }^{\mathrm{u}}$, U. Landgraf ${ }^{\mathrm{e}}$, J.M. Le Goff ${ }^{\mathrm{r}, \mathrm{d}}$, F. Lehar ${ }^{\mathrm{r}}$, A. de Lesquen ${ }^{\mathrm{r}}$, J. Lichtenstadt ${ }^{\mathrm{t}}$, M. Litmaath ${ }^{\mathrm{n}}$, S. Lopez-Ponte ${ }^{\mathrm{s}, \mathrm{h}}$, M. Lowe ${ }^{\mathrm{q}}$, A. Magnon ${ }^{\mathrm{r}}$, G.K. Mallot ${ }^{\mathrm{j}}$, F. Marie ${ }^{\mathrm{r}}$, A. Martin ${ }^{\mathrm{u}}$, J. Martino ${ }^{\mathrm{r}}$, T. Matsuda ${ }^{\mathrm{m}, 3}$, B. Mayes ${ }^{\mathrm{h}}$, J.S. McCarthy ${ }^{\mathrm{w}}$, K. Medved ${ }^{\mathrm{i}}$, G. van Middelkoop ${ }^{\mathrm{n}}$, D. Miller ${ }^{\mathrm{p}}$, K. Mori ${ }^{\mathrm{m}}$, J. Moromisato $^{\circ}$, A. Nagaitsev $^{i}$, J. Nassalski ${ }^{x, d}$, L. Naumann ${ }^{\text {d,24, }}$, T.O. Niinikoski ${ }^{\text {d, }}$, J.E.J. Oberski ${ }^{n}$, A. Ogawa ${ }^{m}$, C. Ozben ${ }^{b}$ A. Penzo ${ }^{\text {, }}$, C. Perez ${ }^{\text {s }}$, F. Perrot-Kunne ${ }^{\mathrm{r}}$, D. Peshekhonov ${ }^{i}$, R. Piegaia ${ }^{y, 8}$, L. Pinsky ${ }^{h}$, S. Platchkov ${ }^{r}$, M. Plo ${ }^{s}$, D. Pose ${ }^{i}$, H. Postma ${ }^{n}$, J. Pretz ${ }^{j}$, T. Pussieux ${ }^{\mathrm{r}}$, J. Pyrlik ${ }^{\mathrm{h}}$, I. Reyhancan ${ }^{\mathrm{b}}$, A. Rijllart ${ }^{\mathrm{d}}$, J.B. Roberts ${ }^{\mathrm{q}}$, S. Rock $^{\mathrm{d}, 9}$, M. Rodriguez ${ }^{\text {s,10, E. Rondio }}{ }^{x}$, I. Sabo ${ }^{t}$, J. Saborido ${ }^{\text {s }}$, A. Sandacz ${ }^{x}$, I. Savin ${ }^{\text {i }}$, P. Schiavon ${ }^{\text {, }}$ R. Segel ${ }^{\text {P }, ~ R . ~ S e i t z ~}{ }^{j, 11}$, Y. Semertzidis ${ }^{d, 12}$, P. Shanahan ${ }^{\text {p.13 }}$, E.P.Sichtermann ${ }^{\text {, }}$, G.I. Smirnov ${ }^{i}$, A. Staude ${ }^{\ell}$, A. Steinmetz ${ }^{j}$, U. Stiegler ${ }^{d}$, H. Stuhrmann ${ }^{f}$, M. Szleper ${ }^{x}$,

F. Tessarotto ${ }^{\mathrm{a}}, \mathrm{M}$. Velasco ${ }^{\mathrm{p}}, \mathrm{J}$. Vogt ${ }^{\ell}, \mathrm{R}$. Voss ${ }^{\mathrm{d}}, \mathrm{R}$. Weinstein ${ }^{\mathrm{h}}, \mathrm{C}$. Whitten ${ }^{\mathrm{c}}$,

R. Windmolders ${ }^{k}$, R. Willumeit ${ }^{\mathrm{f}}$, W. Wislicki ${ }^{\mathrm{x}}$, A. Witzmann ${ }^{\mathrm{e}}$, A.M. Zanetti ${ }^{\mathrm{u}}$, J. Zhao ${ }^{\mathrm{f}}$ a University of Bielefeld, Physics Department, 33501 Bielefeld, Germany ${ }^{14}$

${ }^{\mathrm{b}}$ Bogaziçi University and Cekmece Nuclear Research Center, Istanbul Technical University, Istanbul University, Istanbul. Turkey 15 c University of California, Department of Physics, Los Angeles, 90024 CA, USA ${ }^{16}$ d CERN, 1211 Geneva 23, Switzerland

e University of Freiburg, Physics Department, 79104 Freiburg, Germany ${ }^{14}$ 'GKSS, 21494 Geesthacht, Germany ${ }^{14}$ 
E Helsinki University of Technology, Low Temperature Laboratory and Institute of Particle Physics Technology, Otakaari $3 A, 02150$ Finland

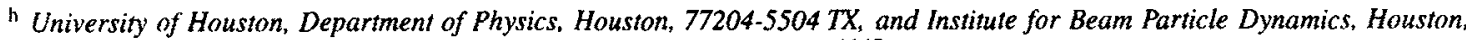
77204-5506 TX, USA ${ }^{16,17}$

i JINR, Laboratory of Particle Physics, Dubna, Russia

j University of Mainz, Institute for Nuclear Physics, 55099 Mainz, Germany ${ }^{14}$

k University of Mons, Faculty of Science, 7000 Mons, Belgium

${ }^{\ell}$ University of Munich, Physics Department, 80799 Munich, Germany ${ }^{14}$

m Nagoya University, Department of Physics, Furo-Cho, Chikusa-Ku, 464 Nagoya, Japan ${ }^{18}$

n NIKHEF, Delft Universify of Technology, FOM and Free University, 1009 AJ Amsterdam. The Netherlands 19

- Northeastern University, Department of Physics, Boston, 02115 MA, USA 17

${ }^{\mathrm{P}}$ Northwestern University, Department of Physics. Evanston, 60208 IL, USA ${ }^{16,17}$

${ }^{\mathrm{q}}$ Rice University, Bonner Laboratory, Houston, 77251-1892 TX, USA ${ }^{16}$

' DAPNIA, C.E. Saclay, 91191 Gif-sur-Yvette, France 20

${ }^{s}$ University of Santiago, Department of Particle Physics, 15706 Santiago de Compostela. Spain ${ }^{21}$

${ }^{1}$ Tel Aviv University, School of Physics, 69978 Tel Aviv, Israel 22

" INFN Trieste and University of Trieste, Department of Physics, 34127 Trieste, Italy

* Uppsala University, Department of Radiation Sciences, 75121 Uppsala, Sweden

"University of Virginia, Department of Physics, Charlottesville, 2290I VA, USA ${ }^{16}$

xoltan Institute for Nuclear Studies and Warsaw University, 00681 Warsaw, Poland ${ }^{23}$

y Yale University, Department of Physics, New Haven, 065II CT, USA ${ }^{16}$

Received 29 June 1995; revised manuscript received 13 July 1995

Editor: L. Montanet

\begin{abstract}
We present a new measurement of the spin-dependent structure function $g_{1}^{\mathrm{d}}$ of the deuteron in deep inelastic scattering of $190 \mathrm{GeV}$ polarised muons on polarised deuterons, in the kinematic range $0.003<x<0.7$ and $1 \mathrm{GeV}^{2}<Q^{2}<60 \mathrm{GeV}^{2}$. This structure function is found to be negative at small $x$. The first moment $\Gamma_{1}^{\mathrm{d}}=\int_{0}^{1} g_{1}^{\mathrm{d}} \mathrm{d} x$ evaluated at $Q_{0}^{2}=10 \mathrm{GeV}$ is $0.034 \pm 0.009$ (stat.) \pm 0.006 (syst.). This value is below the Ellis-Jaffe sum rule prediction by three standard deviations. Using our earlier determination of $\Gamma_{1}^{p}$, we obtain $\Gamma_{1}^{p}-\Gamma_{1}^{n}=0.199 \pm 0.038$ which agrees with the Bjorken sum rule.
\end{abstract}

\footnotetext{
${ }^{1}$ Now at CERN, 1211 Geneva 23, Switzerland.

${ }^{2}$ Now at INFN Trieste, 34127 Trieste, Italy.

${ }^{3}$ Permanent address: Miyazaki University, 88921 Miyazaki-Shi, Japan.

${ }^{4}$ Permanent address: Paul Scherrer Institut, 5232 Villigen, Switzerland.

${ }^{S}$ Research fellow of JSPS.

${ }^{6}$ Permanent address: University of California, Institute of Particle

Physics, Santa Cruz, 95064 CA, USA.

${ }^{7}$ Permanent address: KEK, 305 Ibaraki-Ken, Japan.

${ }^{8}$ Permanent address: University of Buenos Aires. Physics Department, 1428 Buenos Aires, Argentina.

${ }^{9}$ Permanent address: The American University, Washington D.C. 20016, USA.

${ }^{10}$ Now at Uppsala University, 75121 Uppsala, Sweden.

${ }^{11}$ Now at Dresden Technical University, 01062 Dresden, Germany.

12 Permanent address: Brookhaven National Laboratory, Upton, 11973 NY, USA.

${ }^{13}$ Now at Fermi National Accelerator Laboratory, Batavia, 60510
}

Illinois, USA.

${ }^{14}$ Supported by the Bundesministerium für Bildung, Wissenschaft, Forschung und Technologie.

15 Partially supported by TUBITAK and the Centre for TurkishBalkan Physics Research and Application (Bogaziçi University).

${ }^{16}$ Supported by the U.S. Department of Energy.

${ }^{17}$ Supported by the U.S. National Science Foundation.

${ }^{18}$ Supported by Ishida Foundation, Mitsubishi Foundation and Monbusho International Science Research Program.

${ }^{19}$ Supported by the National Science Foundation (NWO) of the Netherlands.

${ }^{20}$ Supported by the Commissariat à l'Ènergie Atomique.

${ }^{21}$ Supported by Comision Interministerial de Ciencia y Tecnologia.

22 Supported by the Israel Science Foundation.

${ }^{23}$ Supported by KBN SPUB/P3/209/94.

${ }^{24}$ Deceased. 
The deuteron and the proton are the simplest and theoretically best understood nuclei to study the internal spin structure of the nucleons. The determination of the first moment $\Gamma_{1}^{\mathrm{d}}=\int_{0}^{1} g_{1}^{\mathrm{d}} \mathrm{d} x$ of the deuteron structure function $g_{1}^{\mathrm{d}}$ allows a test of the Ellis-Jaffe sum rule [1] and can be used to determine the fraction of the nucleon spin carried by quarks. Together with $\Gamma_{1}^{\mathrm{p}}$ it provides a test of the fundamental Bjorken polarisation sum rule [2].

Measurements of the spin-dependent structure function of the deuteron, $g_{1}^{\mathrm{d}}$, were made by the SMC at CERN [3] and by the E143 collaboration at SLAC [4]. Both experiments found deviations from the EllisJaffe prediction. In our previous measurement $g_{1}^{\mathrm{d}}$ was found to be negative for $x<0.03$ within large statistical errors. This suggested that the neutron and the proton spin structure functions are different in this small $x$ region, contrary to the unpolarised ones [5]. In this paper, we report on a new and more precise measurement of $g_{1}^{\mathrm{d}}$, where longitudinally polarised muons of $190 \mathrm{GeV}$ were scattered from longitudinally polarised deuterons in the extended kinematic range $0.003<$ $x<0.7$ and $1 \mathrm{GeV}^{2}<Q^{2}<60 \mathrm{GeV}^{2}$.

The target previously used for the measurement of $g_{1}^{\mathrm{p}}$ [6] was employed with fully deuterated butanol as target material. The two target cells were increased in length to $65 \mathrm{~cm}$, reducing the gap between them to $20 \mathrm{~cm}$. The target cells were polarised in opposite directions by the method of dynamic nuclear polarisation (DNP) using frequency modulated microwaves [7]. The polarisation was measured [8] at ten locations equally spaced along the target by NMR coils connected to series $Q$-meter circuits with an overall accuracy of $\Delta P_{T} / P_{T}=5.4 \%$. Vector polarisations in excess of \pm 0.40 were routinely reached in less than 12 hours of DNP. An average polarisation difference between the upstream and downstream target cells of 0.97 was obtained throughout the data-taking and the highest polarisations reached were +0.51 and -0.61 . The spin directions were reversed every five hours by rotation of the magnetic field direction with a polarisation luss of less than 0.003 .

The $\mu^{+}$beam had an intensity of $4.5 \times 10^{7}$ per spill of $2.4 \mathrm{~s}$ duration with a $14.4 \mathrm{~s}$ period and an average muon momentum of $190 \mathrm{GeV}$. The muon momentum was determined with an accuracy of $\Delta p / p=$ $0.5 \%$ in a magnetic spectrometer upstream of the tar- get. The incident muon track was reconstructed with an angular resolution of $0.1 \mathrm{mrad}$ using scintillator hodoscopes and a proportional chamber. The beam polarisation was $P_{\mu}=-0.811 \pm 0.028$ (3805) (stat.) \pm 0.029 (syst.). It was determined from the shape of the energy spectrum of positrons from the decay $\mu^{+} \rightarrow$ $e^{+} \nu_{e} \bar{\nu}_{\mu}$ [9]. This value is based on the analysis of $15 \%$ of the data collected for the polarisation measurement.

To measure the trajectory of the scattered muon more than 150 planes of proportional chambers, drift chambers and streamer tubes were installed before, behind and inside the forward spectrometer magnet (FSM). The muon was identified as a track behind a $2 \mathrm{~m}$ thick hadron absorber made of iron. On the average, a muon track was reconstructed from $\sim 60$ hits in these detectors. The large number of hits minimises the effect of individual plane inefficiencies on the overall track reconstruction efficiency and thus on the spectrometer acceptance. The angle and the momentum of the scattered muon were determined with average accuracies of $0.4 \mathrm{mrad}$ and $1.3 \mathrm{GeV}$, respectively. The interaction vertex was reconstructed with a resolution better than $30 \mathrm{~mm}(0.3 \mathrm{~mm})$ parallel (perpendicular) to the beam direction. The two trigger systems were based on scintillator hodoscopes and veto counters and are the same as used previously in our measurements with a proton target [6].

The asymmetry $A^{\mathrm{d}}=\left(\sigma^{\uparrow \downarrow}-\sigma^{\uparrow \uparrow}\right) /\left(\sigma^{\uparrow \downarrow}+\sigma^{\uparrow \uparrow}\right)$ of the single-photon exchange cross sections for antiparallel $(\uparrow \downarrow)$ and parallel $(\uparrow \uparrow)$ longitudinal spins of the muon and deuteron and the structure function $g_{1}^{d}$ are related to the virtual-photon deuteron asymmetries $A_{1}^{\mathrm{d}}$ and $A_{2}^{\mathrm{d}}$ by [10]:

$A^{\mathrm{d}}=D\left(A_{1}^{\mathrm{d}}+\eta A_{2}^{\mathrm{d}}\right)$

and

$g_{1}^{\mathrm{d}}=\frac{F_{2}^{\mathrm{d}}}{2 x(1+R)}\left(A_{1}^{\mathrm{d}}+\gamma A_{2}^{\mathrm{d}}\right)$.

Here we neglected the contribution from the quadrupole structure function $b_{1}$ which is cxpected to be small in the kinematic range of our data [11]. The coefficients $\eta, \gamma$ depend only on kinematic variables. The depolarisation factor $D$ depends also on the unpolarised structure function $R$. The virtualphoton deuteron asymmetries are defined as [12] $A_{1}^{\mathrm{d}}=\frac{1}{2}\left(\sigma_{0}^{T}-\sigma_{2}^{T}\right) / \sigma^{T}, A_{2}^{\mathrm{d}}=\frac{1}{2}\left(\sigma_{0}^{T L}+\sigma_{1}^{T L}\right) / \sigma^{T}$ 
where $\sigma^{T}=\frac{1}{2}\left(\sigma_{0}^{T}+\sigma_{2}^{T}\right)$ is the total transverse photoabsorption cross section, $\sigma_{J}^{T}$ is the virtual-photon deuteron absorption cross section for total spin projection $J$ in the direction of the virtual photon and $\sigma_{J}^{T L}$ results from the spin-flip amplitude in forward photon deuteron Compton scattering. For the unpolarised structure functions $F_{2}$ and $R$ we used the parametrisations by the NMC [13] and SLAC [14], respectively, where $R$ is the same for the proton and for the neutron. The average of the free nucleon structure functions is related to the deuteron structure function $g_{1}^{\mathrm{d}}=\frac{1}{2}\left(g_{1}^{\mathrm{p}}+g_{1}^{\mathrm{n}}\right)\left(1-\frac{3}{2} \omega_{D}\right)$. For the probability of the deuteron to be in a D-state we have taken $\omega_{D}=0.05 \pm 0.01$ which covers most of the published values [15]. Off-mass-shell effects in $g_{l}^{\mathrm{d}}$ were calculated [16] to be negligible in the region of our measurement. In this region the factors $\eta$ and $\gamma$ are also small and we neglected the contributions from $A_{2}^{\text {d }}$ to Eqs. (1) and (2). To estimate the resulting systematic error we used an upper limit of

$$
\left|A_{2}^{\mathrm{d}}\right| \leq\left(1-1.5 \omega_{D}\right)\left(A_{2}^{\mathrm{p}}+\frac{F_{2}^{\mathrm{n}}}{F_{2}^{\mathrm{p}}} \sqrt{R}\right) /\left(1+\frac{F_{2}^{\mathrm{n}}}{F_{2}^{\mathrm{p}}}\right)
$$

calculated at the $95 \%$ confidence level. The valuc $A_{2}^{\mathrm{p}}$ was taken from the measurement by the SMC [17], the ratio $F_{2}^{\mathrm{n}} / F_{2}^{\mathrm{p}}$ from the NMC parametrisation [5] and the upper limit for $A_{2}^{\mathrm{n}}$ was taken to be $\sqrt{R}$ [10].

Events were selected by applying cuts which ensured that the beam flux was the same for both target cells and that events from unwanted target material were suppressed. Further cuts removed muons from decays of hadrons and minimised the size of kinematic smearing effects and radiative corrections. In total $6 \times 10^{6}$ events from the new measurement were used in the analysis.

The asymmetry $A_{1}^{\mathrm{d}}$ was extracted from combinations of data sets taken before and after a reversal of the target polarisation [6]. In the evaluation of asymmetries the muon fluxes, the amount of target material and the acceptances for the two target cells cancel. However, the measured asymmetry is sensitive to the time dependence of the ratio $r=\left(n_{u} a_{u}\right) /\left(n_{d} a_{d}\right)$, where $a_{u(d)}$ and $n_{u(d)}$ are the acceptance and the target mass for the upstream (downstream) target cell, respectively. A change of $r$ by $\Delta r$ between target polarisation reversals gives rise to a false asymmetry $\Delta A_{1}^{\mathrm{d}}=(\Delta r / r) /\left(4 f P_{\mu} P_{T} D\right)$. The dilution factor $f \simeq$ 0.20 is the fraction of scattering events from deuterons.

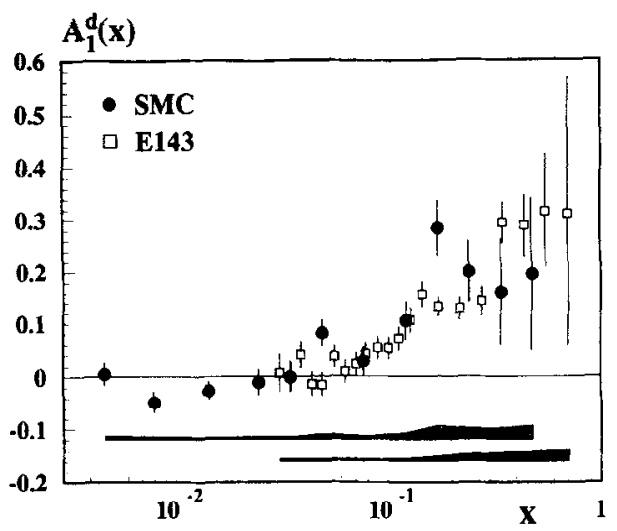

Fig. 1. The virtual-photon deuteron cross section asymmetry $A_{1}^{\mathrm{d}}$ as a function of the scaling variable $x$ at the average $Q^{2}$ of each $x$ bin. Only statistical errors are shown on the data points. The size of the systematic errors is indicated by the shaded areas. Results from the SLAC E143 experiment [4] are shown for comparison.

For each data set we have determined the efficiencies of all tracking detectors. An upper limit of 0.003 for $\left|\Delta A_{1}^{\mathrm{d}}\right|$ was estimated from the largest observed efficiency changes in all detectors between polarisation reversals. Radiative corrections were applied to compute $A_{1}^{\mathrm{d}}$ from the measured asymmetries [18] and they were found to affect $A_{1}^{\mathrm{d}}$ by less than 0.003 in the entire kinematic range.

The results for $A_{1}^{\mathrm{d}}(x)$ at the average $Q^{2}$ of each bin of $x$ are given in Table 1 and are shown in Fig. 1. In the analysis we have included data taken previously with a beam of $100 \mathrm{GeV}$ [3], which have an average $Q^{2}$ smaller by a factor of about two. The results from both measurements are in agreement. The dominant systematic errors are due to uncertainties in $A_{2}^{\mathrm{d}}$, radiative corrections, time dependence of the acceptance ratio, target and beam polarisations and $R$. The total systematic error given in Table 1 and shown in Fig. 1 combines all contributions in quadrature.

The results for $g_{1}^{\mathrm{d}}(x)$ at the average $Q^{2}$ of each $x$ bin are also given in Table 1 and are shown in Fig. 2. Results for $g_{1}^{d}$ evaluated at fixed $Q_{0}^{2}=10 \mathrm{GeV}^{2}$ assuming that $A_{1}^{\mathrm{d}}$ is independent of $Q^{2}$ are included in Table 1 as well. The negative values of $g_{1}^{d}$ at small $x$ reported in our previous publication [3] are confirmed.

The contribution to $\Gamma_{1}^{d}$ from the measured $x$ range is: 
Table 1

Results on the virtual photon deuteron asymmetry $A_{1}^{\mathrm{d}}$, the deuteron structure function $g_{1}^{\mathrm{d}}$, and the neutron structure function $g_{1}^{\mathrm{n}}$ from all SMC data. The first error is statistical, the second one is systematic. For the evaluation of $g_{1}^{\mathrm{d}}\left(Q^{2}=10 \mathrm{GeV}^{2}\right)$, it has been assumed that $A_{1}^{\mathrm{d}}$ does not depend on $Q^{2}$.

\begin{tabular}{|c|c|c|c|c|c|c|c|c|c|c|c|c|}
\hline \multirow{2}{*}{$\frac{x \text { Range }}{0.003-0.006}$} & \multirow{2}{*}{$\frac{\langle x\rangle}{0.005}$} & \multirow{2}{*}{$\frac{\left\langle Q^{2}\right\rangle}{1.3}$} & \multirow{2}{*}{$\frac{A_{1}^{\mathrm{d}}}{0.005 \pm 0.023 \pm 0.007}$} & \multicolumn{3}{|c|}{$g_{1}^{\mathbf{\alpha}}$} & \multicolumn{3}{|c|}{$g_{1}^{\mathrm{n}}$} & \multicolumn{2}{|c|}{$g_{1}^{\mathrm{d}}\left(Q_{0}^{2}=10\right.$} & \multirow{2}{*}{$\begin{array}{l}\left.\mathrm{GeV}^{2}\right) \\
\pm 0.3\end{array}$} \\
\hline & & & & 0.13 & \pm 0.57 & \pm 0.17 & -1.0 & \pm 1.3 & \pm 0.4 & 0.2 & $\perp 1.0$ & \\
\hline $0.006-0.010$ & 0.008 & 2.0 & $-0.049 \pm 0.021 \pm 0.006$ & -0.80 & \pm 0.35 & \pm 0.14 & -2.4 & \pm 0.8 & \pm 0.2 & -1.3 & \pm 0.5 & \pm 0.2 \\
\hline $0.010-0.020$ & 0.014 & 3.2 & $-0.026 \pm 0.018 \pm 0.004$ & -0.26 & \pm 0.18 & \pm 0.06 & -1.0 & \pm 0.4 & \pm 0.1 & -0.35 & \pm 0.24 & \pm 0.07 \\
\hline $0.020-0.030$ & 0.025 & 4.9 & $-0.011 \pm 0.025 \pm 0.005$ & -0.07 & \pm 0.16 & \pm 0.03 & -0.46 & \pm 0.39 & \pm 0.07 & -0.08 & \pm 0.19 & \pm 0.04 \\
\hline $0.030-0.040$ & 0.035 & 6.4 & $-0.001 \pm 0.030 \pm 0.005$ & 0.00 & \pm 0.14 & \pm 0.02 & -0.34 & \pm 0.35 & \pm 0.06 & 0.00 & \pm 0.16 & \pm 0.03 \\
\hline $0.040-0.060$ & 0.049 & 8.5 & $0.082 \pm 0.027 \pm 0.010$ & 0.28 & \pm 0.09 & \pm 0.04 & 0.16 & \pm 0.23 & \pm 0.08 & 0.28 & \pm 0.09 & \pm 0.03 \\
\hline $0.060-0.100$ & 0.077 & 11.9 & $0.029 \pm 0.028 \pm 0.007$ & 0.06 & \pm 0.06 & \pm 0.01 & -0.23 & \pm 0.15 & \pm 0.04 & 0.06 & \pm 0.06 & \pm 0.01 \\
\hline $0.100-0.150$ & 0.12 & 16.8 & $0.106 \pm 0.048 \pm 0.012$ & 0.14 & \pm 0.05 & \pm 0.02 & -0.09 & \pm 0.12 & \pm 0.04 & 0.13 & \pm 0.05 & \pm 0.01 \\
\hline $0.150 \quad 0.200$ & 0.17 & 22.2 & $0.283 \pm 0.055 \pm 0.026$ & 0.24 & \pm 0.05 & \pm 0.03 & 0.25 & \pm 0.12 & \pm 0.05 & 0.23 & \pm 0.05 & \pm 0.02 \\
\hline $0.200-0.300$ & 0.24 & 29.4 & $0.200 \pm 0.060 \pm 0.021$ & 0.10 & \pm 0.03 & \pm 0.01 & 0.06 & \pm 0.08 & \pm 0.03 & 0.10 & \pm 0.03 & \pm 0.01 \\
\hline $0.300-0.400$ & 0.34 & 38.8 & $0.159 \pm 0.103 \pm 0.021$ & 0.040 & \pm 0.026 & $5 \pm 0.006$ & -0.080 & $0 \pm 0.066$ & $6 \pm 0.017$ & 0.043 & $3 \pm 0.028$ & $8 \pm 0.004$ \\
\hline $0.400-0.700$ & 0.48 & 48.7 & $0.195 \pm 0.147 \pm 0.026$ & 0.017 & $7 \pm 0.013$ & \pm 0.002 & -0.024 & $4 \pm 0.034$ & $4 \pm 0.008$ & 0.022 & $2 \pm 0.016$ & $6 \pm 0.002$ \\
\hline
\end{tabular}
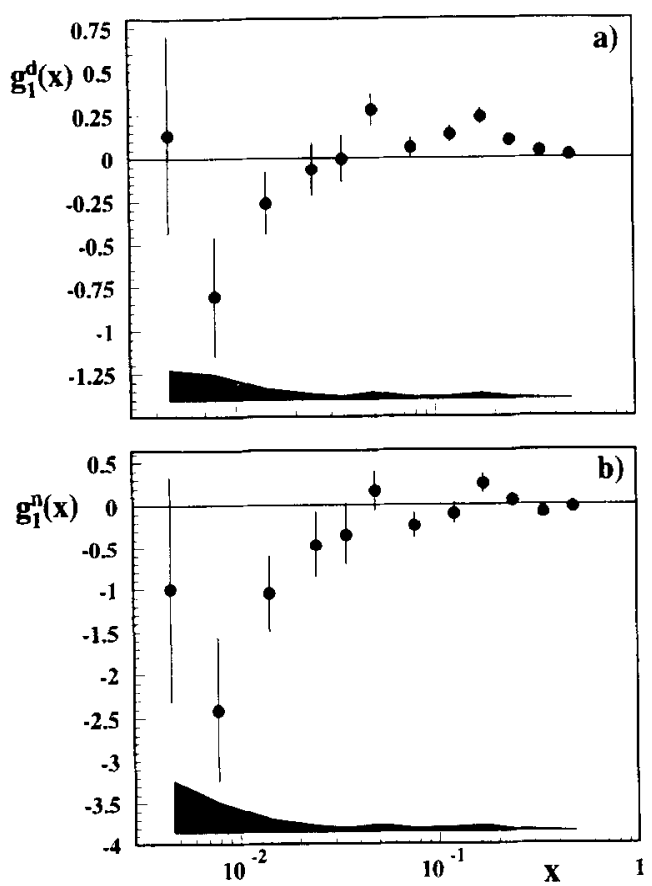

Fig. 2. The spin-dependent structure functions (a) $g_{1}^{\mathrm{d}}(x)$ and (b) $g_{1}^{\prime \prime}(x)$, as a function of the scaling variable $x$ at the average $Q^{2}$ of each $x$ bin. Only statistical errors are shown on the data points. The size of the systematic errors is indicated by the shaded areas.

$$
\begin{aligned}
& \int_{0.003}^{0.7} g_{1}^{\mathrm{d}}\left(x, Q_{0}^{2}\right) \mathrm{d} x=0.0358 \pm 0.0087 \pm 0.0051 \\
& \quad\left(Q_{0}^{2}=10 \mathrm{GeV}^{2}\right)
\end{aligned}
$$

where here and in the following the first error is statistical and the second one is systematic. The contributions from the unmeasured region at small $x$ is $\int_{0}^{0.003} g_{1}^{\mathrm{d}}(x) \mathrm{d} x=-0.0028 \pm 0.0016$. This contribution has been obtained assuming $g_{1}^{\mathrm{d}}(x)=$ constant and fitting the constant to the two lowest $x$ points. The error has been enlarged to 0.0028 to account for cxtrapolations obtained using the general form of Regge dependence $g_{1}^{d}(x) \propto x^{\alpha}$, with $0 \leq \alpha \leq 0.5$ [19]. The contribution from the unmeasured region at large $x$ is $\int_{0.7}^{1} g_{1}^{\mathrm{d}}(x) \mathrm{d} x=0.0006 \pm 0.0010$. For the extrapolation to $x=1$ we used $A_{1}^{\mathrm{d}}=0.4 \pm 0.6$ which is consistent with the data and with the bound $\left|A_{1}^{\mathrm{d}}\right| \leq 1$. The result for the first moment then is:

$$
\Gamma_{1}^{\mathrm{d}}=0.034 \pm 0.009 \pm 0.006 \quad\left(Q_{0}^{2}=10 \mathrm{GeV}^{2}\right) .
$$

Table 2 details the contributions to the systematic error.

The sum $\Gamma_{1}^{\mathrm{p}}+\Gamma_{1}^{\mathrm{n}}=2 \Gamma_{1}^{\mathrm{d}} /\left(1-1.5 \omega_{D}\right)$ can be used to determine the contributions to the nucleon spin from the sum of the quark spins $(\Delta \Sigma)$ and from the strange quark spin $(\Delta s)$. This analysis relies on quark lightcone algebra and on $\mathrm{SU}(3)_{f}$ relations for the axial vector coupling constants within the baryon octet [1]. The QCD corrcctions [20] were calculated using four quark flavours and $\alpha_{s}\left(Q_{0}^{2}=10 \mathrm{GeV}^{2}\right)=0.24 \pm 0.03$, corresponding to $\alpha_{s}\left(m_{Z}^{2}\right)=0.117 \pm 0.005$ [21]. For the SU(3) $f$ coupling constants $F$ and $D$ we used $\left.F+D=g_{A} / g_{V}=1.2573 \pm 0.0028[2]\right]$ and $F / D=$ $0.575 \pm 0.016[22]$. Neglecting possible contributions 
Table 2

Contributions to the error on $\Gamma_{1}^{d}$

\begin{tabular}{ll}
\hline Source of the error & $\Delta \Gamma_{1}^{\mathrm{d}}$ \\
\hline Extrapolation at low $x$ & 0.0028 \\
Neglect of $A_{2}$ & 0.0025 \\
Radiative corrections & 0.0025 \\
Acceptance variation & 0.0020 \\
Beam polarization & 0.0019 \\
Target polarization & 0.0019 \\
Momentum calibration & 0.0014 \\
Uncertainty on $R$ & 0.0013 \\
Uncertainty on $F_{2}$ & 0.0013 \\
Kinematic resolution & 0.0010 \\
Extrapolation at high $x$ & 0.0010 \\
Dilution factor & 0.0009 \\
Proton background & 0.0006 \\
Total systematic error & 0.0063 \\
Statistics & 0.0087 \\
\hline
\end{tabular}

from polarised charm quarks and polarised gluons we obtain

$\Delta \Sigma=\Delta u+\Delta d+\Delta s=0.20 \pm 0.11$,

corresponding to

$\Delta s=-0.12 \pm 0.04$.

These results are inconsistent with the Ellis-Jaffe assumption of $\Delta s=0$ and the resulting prediction of $\Delta \Sigma=3 F-D[1]$ which is $0.579 \pm 0.025$. For the first moment the Ellis-Jaffe sum rule predicts $\left(\Gamma_{1}^{d}\right)_{E J}=$ $0.070 \pm 0.004$ which is three standard deviations above the measured value. The conclusions on $\Delta s$ and on the Ellis-Jaffe sum rule depend on the $\mathrm{SU}(3)_{f}$ symmetry assumption. It has been shown [23] that the value of $\Delta \Sigma$ is insensitive to $\mathrm{SU}(3)_{f}$ breaking effects.

To test the Bjorken sum rule [2] we combine our results for $\Gamma_{1}^{\mathrm{d}}$ (Eq. (4)) and for $\Gamma_{1}^{\mathrm{p}}=0.136 \pm 0.011 \pm$ 0.011 [6] taking into account correlated errors and obtain

$\Gamma_{1}^{\mathrm{p}}-\Gamma_{1}^{\mathrm{n}}=0.199 \pm 0.038 \quad\left(Q_{0}^{2}=10 \mathrm{GeV}^{2}\right)$.

These results also give $\Gamma_{1}^{n}=-0.063 \pm 0.024 \pm 0.013$. The Bjorken sum rule prediction at $Q_{0}^{2}=10 \mathrm{GeV}^{2}$, including perturbative QCD corrections for four quark flavours [24] and up to third order in $\alpha_{s}$, is
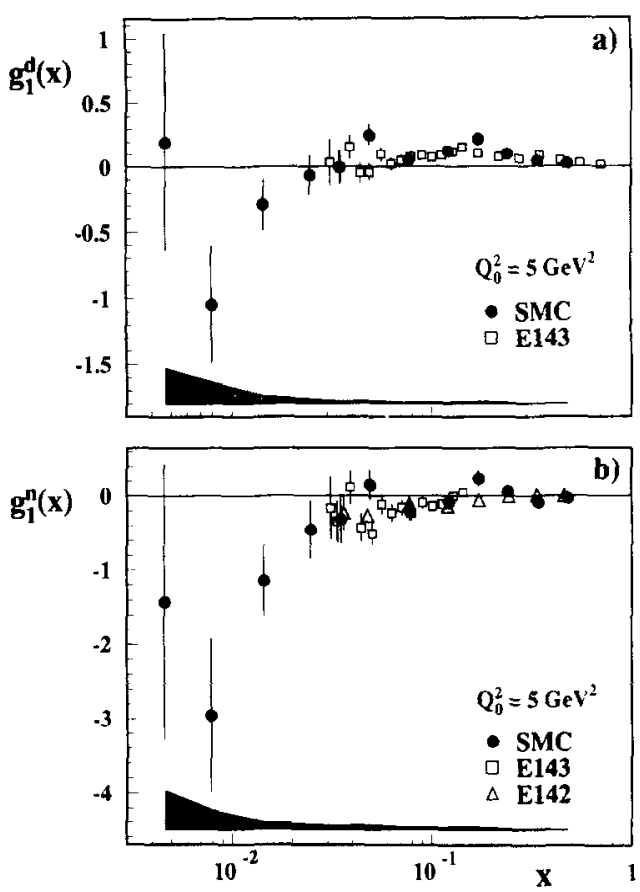

Fig. 3. The spin-dependent structure functions (a) $g_{1}^{\mathrm{d}}(x)$ and (b) $g_{1}^{n}(x)$, as a function of the scaling variable $x$ evaluated at a common $Q_{0}^{2}=5 \mathrm{GeV}^{2}$. Only statistical errors are shown on the data points. Results from the SLAC E142 [25] and E143 [4] experiments are shown for comparison. The size of the systematic errors is indicated by the shaded areas.

$\Gamma_{1}^{\mathrm{p}}-\Gamma_{1}^{\mathrm{n}}=0.187 \pm 0.003$

$$
\text { (Theory, } Q_{0}^{2}=10 \mathrm{GeV}^{2} \text { ) }
$$

in agreement with our result. The error in the prediction is due to uncertainty in $\alpha_{s}$.

Results from this experiment and from the SLAC E143 experiment are compared in Figs. 1 and 3 (a) and are in good agreement. The data from both $\mathrm{cx}$ periments are complementary; the former have higher average $Q^{2}$ and extend to smaller $x$, while the latter have smaller statistical errors. In order to determine $\Gamma_{1}^{d}$ from all available data we combine results on $A_{1}^{\mathrm{d}}$ from both experiments and evaluate $g_{1}^{\mathrm{d}}$ at a common $Q_{0}^{2}=5 \mathrm{GeV}^{2}$, using the parametrisations of $F_{2}^{\mathrm{d}}\left(x, Q^{2}\right)$ and $R\left(x, Q^{2}\right)$ mentioned above. We obtain $\Gamma_{\mathrm{l}}^{\mathrm{d}}=0.031 \pm 0.006$ which gives $\Delta \Sigma=0.18 \pm 0.07$ and $\Delta s=-0.13+0.03$.

The spin-dependent structure function of the neutron, $g_{1}^{\mathrm{n}}$, was determined by combining our results on $g_{1}^{\mathrm{d}}$ and $g_{1}^{\mathrm{p}}[6]$. Results are included in Table 1 and are 
shown in Fig. 2 (b). In Fig. 3 (b) we compare our results to those obtained from the SLAC E143 data [4] and to the results from E142 with a ${ }^{3} \mathrm{He}$ target [25], all evolved to $Q_{0}^{2}=5 \mathrm{GeV}^{2}$. All measurements agree in the common $x$-range. However, our data at small $x$ are more negative than expected from Regge-type extrapolations from the common region. This explains [26] most of the difference between our result for $\Gamma_{1}^{n}$ and that of SLAC E142 [25].

The results on $\Gamma_{1}^{\mathrm{d}}$ presented here were obtained under the assumption that the virtual-photon deuteron asymmetry $A_{1}^{\mathrm{d}}$ does not depend on $Q^{2}$. The same assumption has also been made for all nucleon asymmetries studied so far. Theoretical studies of scale breaking effects have recently been made for spin-dependent structure functions, assuming different polarised quark and gluon distributions $[27-30]$. The predictions are strongly dependent on the assumptions made on the polarised gluon distribution $\Delta g$, which is unknown today. The present accuracy of the data is insufficient to provide meaningful constraints on $\Delta g$.

In summary, we have presented a new and more precise measurement of the spin-dependent structure function $g_{1}^{\mathrm{d}}$ from polarised deep inelastic muondeuteron scattering, extending down to $x=0.003$. We now clearly observe negative values of $g_{1}^{\mathrm{d}}$ at small $x$, in agreement with the trend observed previously. This implies an important difference between the proton and neutron spin structure functions at small $x$. The result for the first moment $\Gamma_{1}^{d}$ disagrees with the EllisJaffe prediction by three standard deviations. The total polarised quark contribution to the nucleon spin is $\Delta \Sigma=0.20 \pm 0.11$. Assuming $S U(3)_{f}$ relations for the coupling constants $F$ and $D$ and measurements of $F+D$ and $F / D$, we find a strange quark contribution $\Delta s=-0.12 \pm 0.04$. Our results for $\Gamma_{1}$ of the proton and the deuteron confirm the Bjorken sum rule.

\section{References}

[1] J. Ellis and R.L. Jaffe, Phys. Rev. D9 (1974) 1444; D10 (1974) 1669.

[2] J.D. Bjorken, Phys. Rev. 148 (1966) 1467; Phys. Rev. DI (1970) 1376.

|3| SMC. B. Adeva et al., Phys. Lett. B302 (1993) 533.
14] SLAC E143, K. Abe et al,, SLAC preprint SLAC-PUB-956734, 1995.

[5] NMC, P. Amaudruz et al., Nucl. Phys. B371 (1992) 3.

[6] SMC, D. Adams et al., Phys. Lett. B329 (1994) 399; Erratum, Phys. Lett. B339 (1994) 332.

17] SMC, B. Adeva et al., CERN preprint CERN-PPE/95-31, 1995.

[8] SMC, B. Adeva et al.. Nucl. Instrum. Methods A349 (1994) 334.

[9] SMC, B. Adeva et al.. Nucl. Instrum. Methods A343 (1994) 363.

[10] V.W. Hughes and J. Kuti, Ann. Rev. Nucl. Part. Sci. 33 (1983) 611 .

[11] H. Khan and P. Hoodbhoy, Phys. Lett. B298 (1993) 181.

[12] P. Hoodbhoy et al., Nucl. Phys. B312 (1989) 571; M.A. Rodriguez, PhD thesis, University of Santiago de Compostela, 1994.

[13] NMC, P. Amaudruz et al., Phys. Lett. B295 (1992) 159 and preprint CERN-PPE/92-124; Errata Oct. 26, 1992 and Apr. 19, 1993.

[14] L.W. Whitlow et al., Phys. Lett. B250 (1990) 193. We use the parametrisation published in L.W. Whitlow, $\mathrm{PhD}$ thesis, Stanford University, 1990.

[15] W. Buck and F. Gross, Phys Rev. D20 (1979) 2361; M.Z. Zuilhof and J.A. Tjon, Phys. Rev. C22 (1980) 2369; M. Lacombe et al., Phys. Rev. C21 (1980) 861; R. Machleidt et al., Phys. Rep. 149 (1987) 1; A.Yu. Umnikov et al., University of Alberta preprint AlbertaThy-29-94, 1994.

[16] L.P. Kaptari et al., Phys. Lett. B321 (1994) 271 ; S.A. Kulagin et al., Institute of Theoretical Physics, Regenshurg University report TPR-94-34, 1994;

[17] SMC, D. Adams et al., Phys. Lett. B336 (1994) 125.

[18] T.V. Kukhto and N.M. Shumeiko, Nucl. Phys. B219 (1983) 412.

[19] R.L. Heimann, Nucl. Phys. B64 (1973) 429; J. Ellis and M. Karliner, Phys. Lett. B213 (1988) 73.

[20] S.A. Larin, Phys. Lett. B334 (1994) 192.

[21] Particle Data Group, L. Montanet et al., Phys. Rev. D50 (1994) 1173.

[22] F.E. Close and R.G. Roberts, Phys. Lett. B316 (1993) 165.

[23] J. Lichtenstadt and H.J. Lipkin, Phys. Lett. B353 (1995) 119.

[24] S.A. Larin and J.A.M. Vermaseren, Phys. Lett. B259 ( 1991 ) 345.

[25] SLAC E142, D.L. Anthony et al., Phys. Rev. Lett. 71 (1993) 959.

[26] SMC, B. Adeva et al., Phys. Lett. B320 (1994) 400.

[27] G. Altarelli, P. Nason and G. Ridolfi, Phys. Lett. B320 (1994) 152.

[281 T. Gehrmann and W.J. Stirling, Z. Phys. C 65 (1995) 461.

[29] E.B. Zijlstra and W.L. van Neerven, Nucl. Phys. B417 (1994) 61

[30] R.D. Ball, S. Forte and G. Ridolfi, CERN report CERNTH/95-31, 1995. 